\title{
Polymorphisms in the uncoupling protein 3 gene and their associations with feed efficiency in chickens
}

\author{
Sihua Jin', Lei Yang ${ }^{1}$, Tingting He ${ }^{1}$, Xinfeng Fan ${ }^{1}$, Yiqiu Wang ${ }^{1}, \mathrm{Kai}_{\mathrm{Ge}}{ }^{1}$, and Zhaoyu Geng ${ }^{1, *}$
}

* Corresponding Author: Zhaoyu Geng Tel: +86-551-6578-5519, Fax: +86-551-6578-6326,

E-mail: gzy@ahau.edu.cn

${ }^{1}$ College of Animal Science and Technology, Anhui Agricultural University, Hefei 230036, China

\section{ORCID}

Zhaoyu Geng

https://orcid.org/0000-0003-1898-7459

Submitted Mar 19, 2018; Revised Apr 22, 2018; Accepted May 29, 2018
Objective: The uncoupling protein 3 (UCP3) is a member of the mitochondrial anion carrier superfamily and has crucial effects on growth and feed efficiency in many species. Therefore, the objective of the present study was to examine the association of polymorphisms in the UCP3 gene with feed efficiency in meat-type chickens.

Methods: Six single nucleotide polymorphisms (SNPs) of the UCP3 gene were chosen to be genotyped using matrix-assisted laser desorption-ionization time-of-flight mass spectrometry in meat-type chicken populations with 724 birds in total. Body weight at 49 (BW49) and 70 days of age (BW70) and feed intake (FI) in the interval were collected, then body weight gain (BWG) and feed conversion ratio (FCR) were calculated individually.

Results: One SNP with a low minor allele frequency $(<1 \%)$ was removed by quality control and data filtering. The results showed that rs13997809 of UCP3 was significantly associated with BWG and FCR $(\mathrm{p}<0.05)$, and that rs13997811 had significant effects on BW70 and BWG $(\mathrm{p}<0.05)$. Rs13997812 of UCP3 was strongly associated with BW70, FI, and FCR ( $<<0.05)$. Furthermore, individuals with AA genotype of rs13997809 had significantly higher BWG and lower FCR $(\mathrm{p}<0.05)$ than those with AT genotype. The GG individuals showed strongly higher BW70 and BWG than AA birds in rs13997811 ( $<<0.05$ ). Birds with the TT genotype of rs13997812 had significantly greater BW70 and lower FCR compared with the CT birds ( $\mathrm{p}<0.05$ ). In addition, the TAC haplotype based on rs13997809, rs13997811, and rs13997812 showed significant effects on BW70, FI, and FCR ( $<<0.05)$.

Conclusion: Our results therefore demonstrate important roles for UCP3 polymorphisms in growth and feed efficiency that might be used in meat-type chicken breeding programs.

Keywords: Uncoupling Protein 3; Polymorphism; Feed Efficiency; Association; Chicken

\section{INTRODUCTION}

In modern poultry production, feed represents approximately $60 \%$ of the total raising costs especially in developing countries. In addition, low feed efficiency chickens produce too much excreta which may result in environment pollution [1]. Therefore, it is imperative for researchers and breeders to select superior feed efficient birds in poultry breeding programs. With the rapid development of whole genome sequencing technologies, molecular and physiological characterization of key candidate genes affecting growth and feed efficiency traits can be used as molecular markers for the genetic improvement of broiler chickens $[2,3]$.

The uncoupling proteins (UCPs) located in the mitochondrial inner membrane are members of anion carrier protein superfamily and regarded as crucial regulators of energy homeostasis [4]. Briefly, UCPs are composed of five isoforms in mammals, including UCP1, UCP2, UCP3, UCP4, and UCP5 [5]. Among these genes, uncoupling protein 3 (UCP3) is mainly expressed in skeletal muscle and plays a vital role in regulating energy metabolism 
at transcriptional and posttranslational levels [6]. In chickens, the UCP3 gene is physically located on chicken chromosome 1 and consists of 6 exons and 5 introns spanning $2.9 \mathrm{kp}$ in the vicinity of quantitative trait loci (QTL) influencing growth and feed conversion ratio (FCR) trait (https://www.animalgenome.org/cgi-bin/QTLdb/GG/index) $[7,8]$.

It is well documented that UCP3 protein decreases metabolic efficiency via uncoupling substrate oxidation in mitochondrial from ATP synthesis by mitochondrial respiration chain, thus dissipating mitochondrial proton gradient by mediating inducible mitochondrial proton leak to regulate energy metabolism [9]. In poultry, the UCP3 gene is mainly expressed in skeletal muscle and plays crucial roles in energy metabolism, limiting reactive oxygen production, fatty acid metabolism, and body weight (BW) $[5,10,11]$. In humans, a missense polymorphism in codon 55 of the UCP 3 gene is significantly associated with obesity susceptibility [12]. It was reported that genetic mutation of g.C4902T in the UCP3 gene is significantly correlated with partial body measurement traits including withers height and chest depth in Chinese Qinchuan cattle [13]. Another study showed that relative expression of UCP3 in the low feed efficiency group is significant higher than that in the high feed efficiency group in beef cattle [14]. In addition, previous research demonstrated that avian UCP mRNA expression in breast muscle of the low feed efficiency chickens is higher than that of high feed efficiency birds [15].

Taken together, numerous studies regarding the relationships between UCP3 gene polymorphisms and growth, feed efficiency and energy metabolism disorders have been performed in mammals and humans [16,17]. Until now, there have been few studies on associations of the UCP3 gene with growth and feed efficiency traits in chickens. The objective of the present study was to investigate the association of polymorphisms in the UCP3 gene with BW, body weight gain (BWG), feed intake (FI), and FCR in meat-type chickens, which may be helpful as molecular markers in future breeding programs to improve growth and feed efficiency of meattype chickens.

\section{MATERIALS AND METHODS}

\section{Ethics statement}

All animal experiments were performed in accordance with the Regulations for the Administration of Affairs Concerning Experimental Animals (Ministry of Science and Technology, China, revised in June 2004) and approved by the Institutional Animal Care and Use Committee of Anhui Agricultural University (permit number: IACUC-20101020). All experimental protocols were carried out according to relevant regulations and recommendations by this committee. All efforts were made to minimize suffering in our birds.

\section{Birds and data collection}

Seven hundred and ninety-six males from the 22nd generation of two yellow meat-type chicken strains were chosen as experimental populations in the present study. These included 335 chickens from N202 strain and 461 birds from N301 strain with both strains having complete pedigree information of three generations $\left(G_{20}\right.$ to $\left.G_{22}\right)$. The two strains were selected based on the appearance, growth and carcass traits within every generation and chosen as valuable genetic sources for a local chicken breeding program. All chickens were derived from a single hatch, wing-banded at day of hatch and raised indoors. All birds were given the same experimental diet (Table 1) provided by Wens Nanfang Poultry Breeding Co. Ltd., Guangdong, China and had the same feeding protocols. Feed and water were freely available during the whole experiment period. At 42 days of age, all birds were transferred into the individual cages with the size of $25 \times 40 \times 45 \mathrm{~cm}$ and special troughs used for recording individual FI. Body weight at 49 (BW49) and 70 (BW70) days of age and FI in the interval were measured individually. The BWG and FCR were calculated according to FI and BW in the interval from 49 to 70 days of age. At the end of the trail, a total of 724 birds ( 439 chicks from N301 and 285 chicks from N202 strain) were used for association analysis of $U C P 3$ polymorphisms with BW49, BW70, FI, BWG, and FCR. However, we removed 72 chickens owing to their incomplete genomic or phenotypic information.

Single nucleotide polymorphisms selection, genotyping and quality control

The single nucleotide polymorphisms (SNPs) in the UCP3 gene were obtained from UCSC Genome Browser database (http://genome.ucsc.edu/cgi-bin/hgGateway). The analysis of all the SNPs was performed by the BLAST program at the NCBI database and DNAMAN 7.0 (Lynnon Biosoft, Quebec, Canada) to guarantee all the SNP sequences were non-homologous associated with other genome sequences. Finally, six SNPs in the UCP3 gene were selected for further analysis.

All blood samples were collected from the wing vein by standard venipuncture and stored in acid citrate dextrose anti-

Table 1. Analyzed nutritional components of the experimental meat-type chicken rations

\begin{tabular}{lc}
\hline Nutrient $^{1)}$ & $\mathbf{4 9 - 7 0 ~ d}$ \\
\hline Metabolizable energy (kcal/kg) & 2,837 \\
Crude protein (\%) & 20.36 \\
Crude fiber (\%) & 4.15 \\
Calcium (\%) & 0.85 \\
Available phosphorus (\%) & 0.63 \\
Methionine (\%) & 0.39 \\
Lysine (\%) & 0.64 \\
1) Feed and analyzed nutrient composition were provide by Wens Nanfang Poultry \\
Breeding Co., Ltd., Guangdong, China.
\end{tabular}


coagulant at $-20^{\circ} \mathrm{C}$ before DNA isolation. Genomic DNA was extracted from whole blood samples using NRBC Blood DNA Kit (Omega, Norcross, GA, USA) in accordance with the manufacturer's procedures. DNA quality was tested by $1.5 \%$ agarose gel electrophoresis and concentration was quantified using the NanoDrop 2000 spectrophotometer (ThermoFisher Scientific, Waltham, MA, USA). The final DNA concentrations were 2 to $10 \mathrm{ng} / \mu \mathrm{L}$ and stored at $-20^{\circ} \mathrm{C}$ for further analyses. Two PCR primers and extension primer were designed using software Assay Design 3.1 (Sequenom, San Diego, CA, USA) for each SNP, as listed in Table 2.

Genotyping of 724 chickens was carried out by matrix-assisted laser desorption-ionization time-of-flight mass spectrometry on the Mass ARRAY iPLEX Platform (Sequenom, USA). Single nucleotide polymorphisms with a genotype call rate $<90 \%$ and minor allele frequency $<1 \%$ were discarded across all birds.

\section{Statistical analysis}

The linkage disequilibrium (LD) between several SNPs in one gene was determined by the Haploview program [18]. Lewontin D' statistic $>0.8$ and squared correlation coefficient $r^{2}>1 / 3$ means sufficiently strong LD [19]. The haplotypes for SNPs in strong LD were inferred using Simwalk2 software (http://watson.hgen.pitt.edu/docs/simwalk2.html). The frequency of SNPs genotypes and Hardy-Weinberg equilibrium were analyzed by the FREQ procedure and chi-square fitness test of SAS version 9.4 (SAS Institute Inc., Cary, NC, USA). The SNPs that deviated from the Hardy-Weinberg equilibrium were removed for further analysis. The association between SNPs or haplotypes and growth, feed efficiency traits were analyzed using the generalized linear mixed model procedure of SAS 9.4 with the following model:

$$
\mathrm{Y}_{\mathrm{ijk}}=\mu+\mathrm{L}_{\mathrm{i}}+\mathrm{G}_{\mathrm{j}}+\mathrm{P}_{\mathrm{k}}+\mathrm{e}_{\mathrm{ijk}}
$$

Where $Y_{\mathrm{ijk}}$ is the observed value of the traits, $\mu$ is the overall population mean, $L_{i}$ is the fixed effect of line, $G_{j}$ is the fixed effect of SNP genotype or haplotype, $\mathrm{P}_{\mathrm{k}}$ is the random effect of family, and $\mathrm{e}_{\mathrm{ijk}}$ is the random error.
Table 2. Primer sequences used in genotyping for the UCP3 gene

\begin{tabular}{|c|c|c|}
\hline SNP & Primer & Sequence $\left(5^{\prime}\right.$ to $\left.3^{\prime}\right)$ \\
\hline \multirow[t]{3}{*}{ rs13997809 } & Forward & ACGTTGGATGGGGACCTTATAGAGACCTAC \\
\hline & Reverse & ACGTTGGATGACCTCAATAAGCTCTCACAC \\
\hline & Extension & GTCACACAGGAGCAGC \\
\hline \multirow[t]{3}{*}{ rs13997810 } & Forward & ACGTTGGATGAATCCCCACCCCTTCACGG \\
\hline & Reverse & ACGTTGGATGTGACACAACCCCAAAGTCCC \\
\hline & Extension & CGCCGCCCTCCCCTCAGAG \\
\hline \multirow[t]{3}{*}{ rs13997811 } & Forward & ACGTTGGATGTGTCCCCACTCGGTGGCAT \\
\hline & Reverse & ACGTTGGATGAAGTGAGGCTGCAGGTAAAG \\
\hline & Extension & CTATTGAGCTCCCACAGCGC \\
\hline \multirow[t]{3}{*}{ rs13997812 } & Forward & ACGTTGGATGAAGTGAGGCTGCAGGTAAAG \\
\hline & Reverse & ACGTTGGATGTGCCCCAGCTGTCCCCACT \\
\hline & Extension & CCCACTCGGTGGCAT \\
\hline \multirow[t]{3}{*}{ rs 1058384613} & Forward & ACGTTGGATGTTGAATGTGAGAGGAGAAGG \\
\hline & Reverse & ACGTTGGATGTCCTCTGTCAATGCCATCTC \\
\hline & Extension & AGCCCCACAATGACA \\
\hline \multirow[t]{3}{*}{ rs315264270 } & Forward & ACGTTGGATGGAGGGGAATGGTTTTTGTGG \\
\hline & Reverse & ACGTTGGATGGTCTGAGAGCTACAATCGTG \\
\hline & Extension & GTTGTGGTTTTGTTTTGTTTTGTT \\
\hline
\end{tabular}

UCP3, uncoupling protein 3; SNP, single nucleotide polymorphisms.

\section{RESULTS AND DISCUSSION}

Previous studies had demonstrated that the UCP3 was a member of the mitochondrial anion carrier protein families that play pivotal roles in energy metabolism and lipid metabolism $[16,17]$. Thus, many researchers had widely examined the possible function of the UCP3 gene in energy homeostasis and its association with growth and feed efficiency in many vertebrates [20,21]. However, there was little published research on associations of UCP3 polymorphisms with growth and feed efficiency traits in chickens. In the present study, six SNPs of the UCP3 gene were chosen and screened in yellow meattype chicken strains. One SNP with a very low minor allele frequency was removed by quality control and data filtering. The remaining five SNPs were further tested as polymorphic with genotype call rate $>90 \%$ and minor allele frequency $>1 \%$ (Table 3).

In the current study, association analysis revealed that three SNPs in the UCP3 gene were significantly associated with

Table 3. Genotypic and allelic frequency of SNPs in UCP3 and Hardy-Weinberg equilibrium test

\begin{tabular}{|c|c|c|c|c|c|c|c|c|}
\hline \multirow{2}{*}{ SNP } & \multirow{2}{*}{ Location } & \multirow{2}{*}{$\begin{array}{c}\text { No. of } \\
\text { chickens }\end{array}$} & \multicolumn{3}{|c|}{ Genotypic frequency } & \multicolumn{2}{|c|}{ Allelic frequency } & \multirow{2}{*}{$\begin{array}{c}\text { p-value for } \\
\text { HWE }\end{array}$} \\
\hline & & & $A A^{1)}$ & $A B$ & BB & A & B & \\
\hline rs13997809 & Promoter & 686 & 0.30 & 0.52 & 0.18 & 0.56 & 0.44 & 0.6485 \\
\hline rs13997811 & Promoter & 703 & 0.30 & 0.46 & 0.24 & 0.53 & 0.47 & 0.3949 \\
\hline rs13997812 & Promoter & 682 & 0.26 & 0.54 & 0.20 & 0.53 & 0.47 & 0.3536 \\
\hline rs13997810 & Promoter & 708 & 0.44 & 0.42 & 0.24 & 0.65 & 0.35 & 0.3363 \\
\hline rs1058384613 & Exon & 654 & 0.30 & 0.44 & 0.26 & 0.52 & 0.48 & 0.1149 \\
\hline rs315264270 & $5^{\prime}$-UTR & 704 & 0.98 & 0.02 & 0 & 0.99 & 0.01 & 0.9655 \\
\hline
\end{tabular}

SNP, single nucleotide polymorphisms; UCP3, uncoupling protein 3; HWE, Hardy-Weinberg Equilibrium test.

1) AA means the dominant allele. 
growth and feed efficiency traits in chickens as listed in Table 4. Rs13997809 of the UCP3 gene was significantly associated with BWG and FCR $(\mathrm{p}<0.05)$, and that rs13997811 was strongly associated with BW at 70 days of age (BW70) and BWG ( $<<$ 0.05). SNP rs13997812 had significant effects on BW70, FI, and FCR $(\mathrm{p}<0.05)$. Furthermore, $\mathrm{LD}$ analysis indicated a high linkage block among rs13997809, rs13997811, and rs13997812 of the UCP3 gene (Supplementary Figure S1) and contributed to $99.81 \%$ of total haplotypes scored. They were H1 (AAC: 48.24\%), H2 (AAT:5.04\%), H3 (AGC:8.63\%), H4 (AGT:7.23\%), H5 (TGC:2.04\%), H6 (TGT:3.56\%), and H7 (TAC:25.07\%), as given in Table 5. Association analysis also showed that the TAC haplotype was significantly associated with BW70, FI, and FCR $(\mathrm{p}<0.05$, Table 5$)$. The results indicated that the UCP3 gene plays an important role in determining growth and feed efficiency traits in our chicken populations. Previous research had demonstrated that an $A / G$ polymorphism in intron 3 of the UCP3 gene is significantly associated with average daily gain, partial efficiency growth, and FCR in beef cattle [21]. It was reported that the UCP3 gene has pivotal roles in uncoupling thermogenesis in beige adipocytes and might be a crucial mediator of thermogenesis in cold-tolerant pigs [22].
Table 5. Haplotypes based on SNPs of rs13997809, rs13997811, and rs13997812 in the UCP3 gene

\begin{tabular}{lccc}
\hline No. & Haplotype & Observations & Frequency (\%) \\
\hline H1 & AAC & 713 & 48.24 \\
H2 & AAT & 75 & 5.04 \\
H3 & AGC & 127 & 8.63 \\
H4 & AGT & 107 & 7.23 \\
H5 & TGC & 30 & 2.04 \\
H6 & TGT & 52 & 3.56 \\
H7 & TAC & 371 & 25.07 \\
\hline
\end{tabular}

SNPs, single nucleotide polymorphisms; UCP3, uncoupling protein 3.

Previous studies demonstrated that the UCP3 gene had crucial effects on energy expenditure and homeostasis, and their polymorphisms were significantly associated with fat metabolism, obesity and diabetes in humans and mice [16,23]. Therefore, it was regarded as an important candidate gene affecting growth and feed efficiency traits via regulating energy balance and lipid metabolism. In this study, individuals with the AA genotype of rs13997809 had larger BWG than those of the AT genotype, whereas FCR of the AA genotype was significant lower than those of the AT genotype $(\mathrm{p}<0.05$, Table

Table 4. Association analysis of UCP3 polymorphisms and haplotypes with growth and feed efficiency traits in meat-type chickens

\begin{tabular}{|c|c|c|c|c|c|c|}
\hline \multirow{2}{*}{$\begin{array}{l}\text { Growth and feed } \\
\text { efficiency traits }\end{array}$} & \multicolumn{6}{|c|}{$p$-value for significant test } \\
\hline & rs13997809 & rs13997810 & rs13997811 & rs13997812 & rs1058384613 & TAC haplotype \\
\hline BW49 & 0.3957 & 0.4394 & 0.1439 & 0.0674 & 0.2466 & 0.2163 \\
\hline BW70 & 0.0832 & 0.9822 & $0.0161^{*}$ & $0.0074^{* *}$ & 0.3049 & $0.0271^{*}$ \\
\hline BWG & $0.0328^{*}$ & 0.2359 & $0.0024^{* *}$ & 0.0684 & 0.9079 & 0.0676 \\
\hline $\mathrm{Fl}$ & 0.0687 & 0.7795 & 0.1124 & $0.0187^{*}$ & 0.7149 & $0.0347^{*}$ \\
\hline FCR & $0.0124^{*}$ & 0.1354 & 0.0510 & $0.0206^{*}$ & 0.5746 & $0.0149^{*}$ \\
\hline
\end{tabular}

UCP3, uncoupling protein 3.

1) BW49, body weight at 49 days of age; BW70, body weight at 70 days of age; BWG, body weight gain from 49 to 70 days of age; Fl, feed intake from 49 to 70 days of age; $F C R$, feed conversion ratio from 49 to 70 days of age.

${ }^{*} p<0.05,{ }^{* *} p<0.01$.

Table 6. Least squares means \pm SE for growth and feed efficiency traits among genotypes of three SNPs in the UCP3 gene in meat-type chickens

\begin{tabular}{|c|c|c|c|c|c|c|}
\hline \multirow{2}{*}{ SNP } & \multirow{2}{*}{ Genotype } & \multicolumn{5}{|c|}{ Growth and feed efficiency traits ${ }^{11}$} \\
\hline & & BW49 (g) & BW70 (g) & BWG (g) & $\mathrm{Fl}(\mathrm{g})$ & FCR \\
\hline \multirow[t]{2}{*}{ rs13997809 } & $\mathrm{AA}(206)$ & $1,101.02 \pm 4.32$ & $1,712.55 \pm 4.40$ & $611.35 \pm 3.53^{\mathrm{a}}$ & $1,770.45 \pm 7.17$ & $2.89 \pm 0.02^{\mathrm{a}}$ \\
\hline & TT(124) & $1,116.20 \pm 2.35$ & $1,720.21 \pm 4.58$ & $604.21 \pm 3.78^{\mathrm{ab}}$ & $1,774.15 \pm 6.47$ & $2.92 \pm 0.02^{\mathrm{ab}}$ \\
\hline \multirow[t]{2}{*}{ rs13997811 } & $\mathrm{GG}(208)$ & $1,110.93 \pm 4.13$ & $1,720.37 \pm 4.65^{A}$ & $610.23 \pm 3.41^{a}$ & $1,786.46 \pm 5.75$ & $2.93 \pm 0.04$ \\
\hline & $\mathrm{GA}(325)$ & $1,118.34 \pm 2.38$ & $1,713.27 \pm 4.31^{\mathrm{A}}$ & $595.84 \pm 2.74^{b}$ & $1,785.31 \pm 6.35$ & $2.99 \pm 0.02$ \\
\hline \multirow[t]{3}{*}{ rs13997812 } & $C C(180)$ & $1,108.45 \pm 4.19$ & $1,716.15 \pm 3.57^{\mathrm{ab}}$ & $608.54 \pm 3.64$ & $1,775.99 \pm 6.63^{\mathrm{a}}$ & $2.92 \pm 0.02^{\mathrm{AB}}$ \\
\hline & CT(366) & $1,112.43 \pm 3.83$ & $1,708.69 \pm 4.63^{\mathrm{a}}$ & $600.54 \pm 2.89$ & $1,770.49 \pm 4.75^{\mathrm{ab}}$ & $2.94 \pm 0.01^{\mathrm{A}}$ \\
\hline & ТT(136) & $1,113.29 \pm 4.25$ & $1,722.65 \pm 3.89^{b}$ & $609.03 \pm 3.75$ & $1,763.68 \pm 7.62^{b}$ & $2.89 \pm 0.03^{B}$ \\
\hline
\end{tabular}

SE, standard error; SNPS, single nucleotide polymorphisms; UCP3, uncoupling protein 3.

1) BW49, body weight at 49 days of age; BW70, body weight at 70 days of age; BWG, body weight gain from 49 to 70 days of age; Fl, feed intake from 49 to 90 days of age; $F C R$, feed conversion ratio in the interval.

${ }^{a-c}$ Among genotypes within each SNP for each trait, means within a row with no common superscript differ $(p<0.05)$.

${ }^{A-C}$ Among genotypes within each SNP for each trait, means within a row with no common superscript differ $(p<0.01)$. 
6). Chickens with the GG genotype of rs13997811 showed strongly higher BW70 and BWG than those of the AA genotype ( $<<0.05$, Table 6). For rs13997812, the TT individuals had significantly higher BW70 and lower FCR compared with the CT birds $(\mathrm{p}<0.05$, Table 6$)$. It was reported that polymorphisms in the UCP3 gene were significantly associated with total cholesterol levels and genetic mutation in intron 1 may be correlated with relative expression levels of the UCP 3 gene in dogs [24]. In humans, recent studies reported that polymorphism of C55T in the UCP3 gene might be associated with obesity [16]. Another study showed that UCP3/BglIpolymorphism was significantly associated with growth traits, and genotype $\mathrm{AB}$ was superior over $\mathrm{AA}$ or $\mathrm{BB}$ genotypes in Nanyang cattle [25]. Therefore, the present study provided important reference for the effects of UCP3 in chicken growth and feed efficiency.

In summary, three SNPs and the TAC haplotype of the $U C P 3$ gene were significantly associated with some growth and feed efficiency traits, which might be used as potential genetic markers in yellow meat-type chicken breeding programs. Further studies are necessary to properly investigate associations of UCP3 polymorphisms with feed efficiency traits in large populations with different chicken strains. Additionally, it is necessary to further investigate the molecular mechanisms of identified SNPs or haplotypes of the UCP3 gene contributing to growth and feed efficiency of chickens.

\section{CONFLICT OF INTEREST}

We certify that there is no conflict of interest with any financial organization regarding the material discussed in the manuscript.

\section{ACKNOWLEDGMENTS}

We would like to thank Drs. Dexiang Zhang and Congliang Ji at Guangdong Wens Nanfang Poultry Breeding Co., Ltd., China for providing chicken samples. This work was supported in part by grants from Natural Science Foundation of Anhui Province (1808085QC61), Key Science and Technology Program of Anhui Province (1704a07020091), The Open Fund of Anhui Provincial Key Laboratory of Local Animal Genetic Resources Conservation and Bio-breeding (AKLGRCB2017008), and Starting Foundation for Young Scientists of Anhui Agricultural University (yj2017-03).

\section{REFERENCES}

1. de Verdal H, Narcy A, Bastianelli D, et al. Improving the efficiency of feed utilization in poultry by selection. 2 . Genetic parameters of excretion traits and correlations with anatomy of the gastro-intestinal tract and digestive efficiency. BMC
Genet 2011;12:71.

2. Diaz-Sanchez S, Hanning I, Pendleton S, D'Souza D. Nextgeneration sequencing: the future of molecular genetics in poultry production and food safety. Poult Sci 2013;92:562-72.

3. Niemann H, Kuhla B, Flachowsky G. Perspectives for feedefficient animal production. J Anim Sci 2011;89:4344-63.

4. Fisler JS, Warden $\mathrm{CH}$. Uncoupling proteins, dietary fat and the metabolic syndrome. Nutr Metab 2006;3:38.

5. Woyda-Ploszczyca AM, Jarmuszkiewicz W. The conserved regulation of mitochondrial uncoupling proteins: From unicellular eukaryotes to mammals. Biochim Biophys Acta 2017; 1858:21-33.

6. Bugge A, Siersbaek M, Madsen MS, et al. A novel intronic peroxisome proliferator-activated receptor gamma enhancer in the uncoupling protein (UCP) 3 gene as a regulator of both UCP2 and -3 expression in adipocytes. J Biol Chem 2010;285: 17310-7.

7. Li Q, Xu Z, Liu L, et al. Effects of breeds and dietary protein levels on the growth performance, energy expenditure and expression of avUCP mRNA in chickens. Mol Biol Rep 2013; 40:2769-79.

8. Toyomizu M, Kikusato M, Kawabata Y, et al. Meat-type chickens have a higher efficiency of mitochondrial oxidative phosphorylation than laying-type chickens. Comp Biochem Physiol A Mol Integr Physiol 2011;159:75-81.

9. Azzu V, Brand MD. The on-off switches of the mitochondrial uncoupling proteins. Trends Biochem Sci 2010;35:298-307.

10. MacLellan JD, Gerrits MF, Gowing A, et al. Physiological increases in uncoupling protein 3 augment fatty acid oxidation and decrease reactive oxygen species production without uncoupling respiration in muscle cells. Diabetes 2005;54:234350.

11.Joubert R, Metayer-Coustard S, Crochet S, et al. Regulation of the expression of the avian uncoupling protein 3 by isoproterenol and fatty acids in chick myoblasts: possible involvement of AMPK and PPARalpha? Am J Physiol Regul Integr Comp Physiol 2011;301:R201-8.

12. Qian L, Xu K, Xu X, et al. UCP2 -866G/A, Ala55Val and UCP3 $-55 \mathrm{C} / \mathrm{T}$ polymorphisms in association with obesity susceptibility - a meta-analysis study. PLoS One 2013;8:e58939.

13. Wang Y, Yang W, Gui L, Wang H, Zan L. Association and expression analyses of the Ucp2 and Ucp3 gene polymorphisms with body measurement and meat quality traits in Qinchuan cattle. J Genet 2016;95:939-46.

14. Kelly AK, Waters SM, McGee M, et al. mRNA expression of genes regulating oxidative phosphorylation in the muscle of beef cattle divergently ranked on residual feed intake. Physiol Genomics 2011;43:12-23.

15. Ojano-Dirain C, Toyomizu M, Wing T, Cooper M, Bottje WG. Gene expression in breast muscle and duodenum from low and high feed efficient broilers. Poult Sci 2007;86:372-81.

16. Brondani LA, de Souza BM, Assmann TS, et al. Association of 
the UCP polymorphisms with susceptibility to obesity: casecontrol study and meta-analysis. Mol Biol Rep 2014;41:505367.

17.Li H, Brahi OH, Zhao X, Xu N, Zhao X. Association of pig UCP3 gene mutations and back fat thickness in the sixth and seventh rib. Mol Biol Rep 2012;39:1823-9.

18. Barrett JC, Fry B, Maller J, Daly MJ. Haploview: analysis and visualization of $\mathrm{LD}$ and haplotype maps. Bioinformatics 2005; 21:263-5.

19. Ardlie KG, Kruglyak L, Seielstad M. Patterns of linkage disequilibrium in the human genome. Nat Rev Genet 2002;3:299-309.

20. Guigal N, Rodriguez M, Cooper RN, et al. Uncoupling protein-3 (UCP3) mRNA expression in reconstituted human muscle after myoblast transplantation in RAG2-/-/gamma c/C5(-) immunodeficient mice. J Biol Chem 2002;277:47407-11.

21. Sherman EL, Nkrumah JD, Murdoch BM, et al. Polymorphisms and haplotypes in the bovine neuropeptide $\mathrm{Y}$, growth hormone receptor, ghrelin, insulin-like growth factor 2 , and uncoupling proteins 2 and 3 genes and their associations with measures of growth, performance, feed efficiency, and carcass merit in beef cattle. J Anim Sci 2008;86:1-16.

22. Lin J, Cao C, Tao C, et al. Cold adaptation in pigs depends on UCP3 in beige adipocytes. J Mol Cell Biol 2017;9:364-75.

23. Jia JJ, Zhang $X, \mathrm{Ge} C R$, Jois $\mathrm{M}$. The polymorphisms of UCP2 and UCP3 genes associated with fat metabolism, obesity and diabetes. Obes Rev 2009;10:519-26.

24. Udagawa C, Tada N, Asano J, et al. The genetic association study between polymorphisms in uncoupling protein 2 and uncoupling protein 3 and metabolic data in dogs. BMC Res Notes 2014;7:904.

25.Zhang R, Li X. Association between IGF-IR, m-calpain and UCP-3 gene polymorphisms and growth traits in Nanyang cattle. Mol Biol Rep 2011;38:2179-84. 